\title{
Testing Modified Gravity theory (MOG) with Type Ia Supernovae, Cosmic Chronometers and Baryon Acoustic Oscillations
}

\author{
Carolina Negrelli, ${ }^{1,2}$ Lucila Kraiselburd, ${ }^{1,2}$ Susana Landau, ${ }^{3,2}$ and Claudia G. Scóccola ${ }^{1,2}$ \\ ${ }^{1}$ Grupo de Astrofísica, Relatividad y Cosmología, \\ Facultad de Ciencias Astronómicas y Geofísicas, Universidad Nacional de La Plata, \\ Paseo del Bosque S/N 1900 La Plata, Argentina \\ ${ }^{2}$ CONICET, Godoy Cruz 2290, 1425 Ciudad Autónoma de Buenos Aires, Argentina \\ ${ }^{3}$ Departamento de Física and IFIBA, Facultad de Ciencias Exactas y Naturales, \\ Universidad de Buenos Aires, Ciudad Universitaria - Pab. I, Buenos Aires 1428, Argentina
}

(Dated: July 9, 2020)

\begin{abstract}
We analyse the MOdified Gravity (MOG) theory, proposed by Moffat, in a cosmological context. We use data from Type Ia Supernovae (SNe Ia), Baryon Acoustic Oscillations (BAO) and Cosmic Chronometers $(\mathrm{CC})$ to test MOG predictions. For this, we perform $\chi^{2}$ tests considering fixed values of $H_{0}$ and $V_{G}$, the self-interaction potential of one of the scalar fields in the theory. Our results show that the MOG theory is in agreement with all data sets for some particular values of $H_{0}$ and $V_{G}$, being the BAO data set the most powerful tool to test MOG predictions, due to its constraining power.
\end{abstract}

\section{INTRODUCTION}

The failure of Newton's theory of Gravitation to successfully predict the motion of stars within galaxies and of galaxies in galaxy clusters was first reported by Oort [1] and Zwicky [2]. Later observations of rotation curves in spiral galaxies [3, 4] also pointed towards the same direction, namely: by assuming General Relativity (or its Newtonian counterpart) as the theory of gravity, the visible matter cannot account for the shape of the rotation curves. To solve this discrepancy, a component of nonluminous matter has been postulated. More recently, observations of astrophysical objects over a large range of mass and spatial scales (such as, for example, galaxy clusters, spiral and dwarf galaxies) [5, 6, 7, 8, 9, 10, 11, 12 have also shown the need for including a dark component of matter if the assumption of General Relativity as the theory of gravity holds.

As a consequence, one of the main ingredients of the standard cosmological model $(\Lambda \mathrm{CDM})$ is a component of matter that does not couple to the electromagnetic field but can be detected via its gravitational interactions. In this way, this model is able to explain type Ia Supernovae data [13, the Cosmic Microwave Background (CMB) power spectra 14, data from Baryon Acoustic Oscillations [15, 16, 17] as well as the formation of large scale structures [18]. However, there is something missing in this picture, namely, the nature of this dark matter is currently unknown and none of the proposed candidates has been detected yet in the laboratory, despite numerous dedicated experimental efforts 19 .

An alternative to explain the mismatch between the data and the predictions of the current theory of gravity relies on a modification of the latter. In this regard, a first attempt was introduced by Milgrom in 1983 [20]; the Modified Newtonian Dynamics (MOND) is a phenomenological proposal that follows from observations of galaxy rotation curves and the Tully-Fisher relation.
More recently, in 2004, Bekenstein proposed a relativistic version of this theory named TeVeS 21 . One of the main unsolved issues of this theory is that it is not able to explain simultaneously the rotation curve of galaxies and the strong lensing effect, as well as the observations of the Bullet cluster [22]. Finally, in 2006, Moffat formulated the MOdified Gravity (MOG) theory in which one massive vector field and three scalar fields are added to the gravitational sector of the theory [23. Moreover, this theory can predict successfully the motion within globular clusters, and clusters of galaxies [24, 25, 26] as well as the rotation curves of spiral and dwarf galaxies [27, 28. Nevertheless, some of us recently showed that the MOG theory is not able to explain the observed rotation curve of the Milky Way 29. Moreover, when analyzing the Bullet cluster data [30, there are different claims in the literature: some authors affirm that MOG cannot account for them [22] while others hold that its predictions can fit data from both the Bullet and the Train Wreck merging clusters [31, 32. Additionally, it has been suggested that this theory is not compatible with the gas profile obtained from X-ray measurements and the strong-lensing properties of well-known galaxy clusters [33. However, in Ref [26] the authors show the opposite. On the other hand, as a consequence of the detection of a neutron star merger followed by its electromagnetic counterpart by the LIGO experiment, theories where the difference between the velocity of gravitational waves and the speed of light is significant, are ruled out 34. However, Green et al. [35] pointed out that this is the case for bi-metric theories such as MOND and TeVeS, but not for MOG. In summary, the controversy about the compatibility of the MOG theory predictions with observational data at different scales is not yet settled.

On the other hand, it is well known that type Ia supernovae data have led to the discovery of the current accelerated expansion of the Universe [36]. However, there is no agreement within the scientific community about 
the physical mechanism responsible for this phenomenon. The simplest candidate is the one adopted by the $\Lambda \mathrm{CDM}$ model, namely, to include a cosmological constant in Einstein equations. Other proposals involve adding extra degrees of freedom to the Standard Model of Particles 37 . Alternatively, the extra degrees of freedom can be added to the gravitational sector of the theory. In this regard, several alternative theories of gravity have been considered 38, being MOG a particular case of such kind of theories. In summary, MOG offers an alternative to both the dark matter and dark energy ingredients of the standard cosmological model.

In this work, we focus on the predictions of the MOG theory on cosmological scales. In order to test its predictions, we consider data from Supernovae type Ia, Baryon Acoustic Oscillations and Cosmic Chronometers. We use $\chi^{2}$ tests to carry out the comparison between the mentioned data sets and the theoretical predictions. For this, we consider two fixed values $H_{0}$ (the one inferred from local data and the one obtained from the CMB data) and several values of $V_{G}$, the self-interaction potential of the scalar field that represents the gravitational constant.

In Section [II] we briefly characterize the main aspects of the MOG theory at cosmological scales, analyzing the Friedmann equations and the expressions of the scalar fields of the theory. In Section III] we describe in detail the data sets that are used to test the predictions of the theory. Results of the comparison between the predictions of the MOG theory and each data set considered in this paper are shown and discussed in Section IV] Finally we present our conclusions in Section $\mathrm{V}$.

\section{COSMOLOGICAL BACKGROUND EVOLUTION OF THE MOG THEORY}

In this section we summarize the main aspects of the MOG theory. In order to account for the effects that in the standard cosmological model are produced by dark matter and dark energy, this theory introduces a massive vector field $\phi^{\mu}$ and three scalar fields which are the following: i) $G$, the gravitational coupling strength, which is promoted to a scalar field; ii) $\mu$, which corresponds to the mass of the vector field; and iii) $\omega$, which describes the coupling strength between the vector field and matter. The gravitational and vector field $\phi^{\mu}$ actions are characterized by:

$$
\begin{aligned}
S_{G} & =\frac{1}{16 \pi} \int \frac{1}{G}(R+2 \Lambda) \sqrt{-g} d^{4} x, \\
S_{\phi} & =\frac{1}{4 \pi} \int \omega\left[\frac{1}{4} B^{\mu \nu} B_{\mu \nu}-\frac{1}{2} \mu^{2} \phi_{\mu} \phi^{\mu}\right. \\
& \left.+V_{\phi}\left(\phi_{\mu} \phi^{\mu}\right)\right] \sqrt{-g} d^{4} x,
\end{aligned}
$$

while the scalar fields action is given by:

$$
\begin{aligned}
S_{S}=\int \frac{1}{G} & {\left[\frac{1}{2} g^{\alpha \beta}\left(\frac{\nabla_{\alpha} G \nabla_{\beta} G}{G^{2}}+\frac{\nabla_{\alpha} \mu \nabla_{\beta} \mu}{\mu^{2}}+\nabla_{\alpha} \omega \nabla_{\beta} \omega\right)\right.} \\
+ & \left.\frac{V_{G}(G)}{G^{2}}+\frac{V_{\mu}(\mu)}{\mu^{2}}+V_{\omega}(\omega)\right] \sqrt{-g} d^{4} x,
\end{aligned}
$$

being $B_{\mu \nu}$ the Faraday tensor of the vector field $\left(B_{\mu \nu}=\right.$ $\left.\partial_{\mu} \phi_{\nu}-\partial_{\nu} \phi_{\mu}\right)$ and $V_{\phi}\left(\phi_{\mu} \phi^{\mu}\right), V_{G}(G), V_{\omega}(\omega)$ and $V_{\mu}(\mu)$, the self-interaction potentials associated with the vector field and the scalar fields. Finally, $\nabla_{\nu}$ is the covariant derivative with respect to the metric $g_{\mu \nu}$.

The Friedmann equations for this theory can be obtained assuming an homogeneous and isotropic spacetime which can be described by Friedmann-LemaîtreRobertson-Walker (FLRW) line element $\left(d s^{2}=d t^{2}-\right.$ $\left.a^{2}(t)\left[\left(1-k r^{2}\right)^{-1} d r^{2}+r^{2} d \Omega^{2}\right]\right)[39$ :

$$
\begin{gathered}
H^{2}+\frac{k}{a^{2}}=\frac{8 \pi G \rho}{3}-\frac{4 \pi}{3}\left(\frac{\dot{G}^{2}}{G^{2}}+\frac{\dot{\mu}^{2}}{\mu^{2}}+\dot{\omega}^{2}-\frac{1}{4 \pi} G \omega \mu^{2} \phi_{0}^{2}\right) \\
+\frac{8 \pi}{3}\left(\frac{\omega G V_{\phi}}{4 \pi}+\frac{V_{G}}{G^{2}}+\frac{V_{\mu}}{\mu^{2}}+V_{\omega}\right)+\frac{\Lambda}{3}+H \frac{\dot{G}}{G}, \quad(4) \\
\frac{\ddot{a}}{a}=-\frac{4 \pi G}{3}(\rho+3 p)+\frac{8 \pi}{3}\left(\frac{\dot{G}^{2}}{G^{2}}+\frac{\dot{\mu}^{2}}{\mu^{2}}+\dot{\omega}^{2}-\frac{1}{4 \pi} G \omega \mu^{2} \phi_{0}^{2}\right) \\
+\frac{8 \pi}{3}\left(\frac{\omega G V_{\phi}}{4 \pi}+\frac{V_{G}}{G^{2}}+\frac{V_{\mu}}{\mu^{2}}+V_{\omega}\right)+\frac{\Lambda}{3} \\
+H \frac{\dot{G}}{2 G}+\frac{\ddot{G}}{2 G}-\frac{\dot{G}^{2}}{G^{2}},
\end{gathered}
$$

these equations can be combined to obtain a differential equation for the scale factor $a$ :

$$
\begin{aligned}
\frac{\ddot{a}}{a}+\frac{\dot{a}^{2}}{2 a^{2}}+\frac{k}{2 a^{2}}=-4 & \pi G p+2 \pi\left(\frac{\dot{G}^{2}}{G^{2}}+\frac{\dot{\mu}^{2}}{\mu^{2}}+\dot{\omega}^{2}-\frac{G \omega \mu^{2} \phi_{0}^{2}}{4 \pi}\right) \\
+ & 4 \pi\left(\frac{\omega G V_{\phi}}{4 \pi}+\frac{V_{G}}{G^{2}}+\frac{V_{\mu}}{\mu^{2}}+V_{\omega}\right) \\
+ & \frac{\Lambda}{2}+\frac{\ddot{G}}{2 G}-\frac{\dot{G}^{2}}{G^{2}}+H \frac{\dot{G}}{2 G} .
\end{aligned}
$$

In this work, like in the standard cosmological model, we model the baryonic matter as a pressureless dust such that $p=0$ and $w=p / \rho=0$. On the other hand, it has been discussed in Section [1 that in the MOG theory it is not necessary to include dark matter to explain astrophysical and cosmological observations. Therefore, only baryonic matter will be included in the modified Friedmann Equations. However, given that the matter density does not appear in Eq. 6, the evolution of the cosmic scale factor and, in consequence, of the Hubble parameter will depend only on the pressure of the baryonic matter which in the case of pressureless dust is zero.

On the other hand, the equation of motion for the scalar fields can be derived from Eq. 3 


$$
\begin{gathered}
\ddot{G} \quad+3 H \dot{G}-\frac{3}{2} \frac{\dot{G}^{2}}{G}+\frac{G}{2}\left(\frac{\dot{\mu}^{2}}{\mu^{2}}+\dot{\omega}^{2}\right)-\frac{3}{G} V_{G}-V_{G}^{\prime} \\
+G\left[\frac{V_{\mu}}{\mu^{2}}+V_{\omega}\right]+\frac{G}{8 \pi} \Lambda-\frac{3 G}{8 \pi}\left(\frac{\ddot{a}}{a}+H^{2}+\frac{k}{a^{2}}\right)=0, \\
\ddot{\mu}+3 H \dot{\mu}-\frac{\dot{\mu}^{2}}{\mu}-\frac{\dot{G}}{G} \dot{\mu}+\frac{G \omega \mu^{3} \phi_{0}^{2}}{4 \pi}+\frac{2}{\mu} V_{\mu}-V_{\mu}^{\prime}=0, \\
\ddot{\omega}+3 H \dot{\omega}-\frac{\dot{G}}{G} \dot{\omega}+\frac{1}{8 \pi} G \mu^{2} \phi_{0}^{2}-\frac{G V_{\phi}}{4 \pi}-V_{\omega}^{\prime}=0,
\end{gathered}
$$

where $V_{G}^{\prime}=\frac{d V_{G}}{d G}, V_{\mu}^{\prime}=\frac{d V_{\mu}}{d \mu}$ and $V_{\omega}^{\prime}=\frac{d V_{\omega}}{d \omega}$.

In Section [1] we pointed out that the MOG theory might explain the late acceleration in the expansion of the Universe, even if Einstein's cosmological constant is set to 0 . The reason for this is that the equation of state associated to the self-interaction potentials $V_{G}, V_{\omega}, V_{\phi}$ and $V_{\mu}$ is of the form $w_{V}=-1$ [40, 41]. One of the goals of this work is to test if the MOG theory can supply the role of the dark energy, therefore we set $\Lambda=0$. On the other hand, as in the standard cosmological model, we consider a flat universe, i.e. $k=0$.

As regards the self-interaction potentials, the most simple assumption would be to take them all equal to 0 . However, it has been shown that in order to describe properly cosmological quantities such as the age and evolutionary stages of the Universe [42, 43, and redshift space distortion (RSD) data [44, $V_{G}$ must be different from 0 . Furthermore, previous studies of the MOG theory in the cosmological context [40, 41] consider $V_{G}>11$. Therefore, in this paper, we consider MOG models with $V_{G}=0$ and $V_{G} \neq 0$ to test different data sets in the cosmological context (see section IV). Also, we set $V_{\mu}=V_{\omega}=V_{\phi}=0$.

To find the expressions for $H(z), G(z), \mu(z)$ and $\omega(z)$, equations (6), (7), (8) and (9) need to be solved numerically with suitable initial conditions. We use the ones proposed in Ref. [40]:

$$
\begin{aligned}
t_{0} & =13.7 \times 10^{9} \text { years, } \\
a_{0} & =c t_{0}, \\
G_{0} & =6 G_{N}, \\
\mu_{0} & =a_{0}^{-1} \\
\omega_{0} & =1 / \sqrt{12}, \\
\phi_{0} & =0 \\
\dot{a}_{0} & =H_{0} a_{0}, \\
\dot{G}_{0}=\dot{\mu}_{0}=\dot{\omega}_{0} & =0
\end{aligned}
$$

where $G_{N}$ refers to the Newton's gravitational constant. The choices of $t_{0}$ and $a_{0}$, are the same as in the flat

\footnotetext{
${ }^{1}$ The case $V_{G}>0$ has not been analyzed in Refs. 43, 44
}

standard cosmological model. Besides, since there is no agreement within the scientific community about the reported value of $H_{0}$ (which is referred in the literature as the $H_{0}$ tension), we consider two different values for this parameter, namely the values inferred from local and CMB observations (see section IV for a detailed discussion of this choice). The solution of the MOG field equations in the spherically symmetric case (for details, see [39, 41]) defines the scalar fields values, being $\mu_{0}$ the inverse of the scale of the Universe. On the other hand, the choice of $G_{0}$ is motivated by the following reason: an effective gravitational constant $G_{\text {eff }} \approx 6 G_{N}$ at the Yukawa distance $r=\mu^{-1}$ is compatible with the results coming from the test particle equation of motion expression, being $G_{\text {eff }} \approx 20 G_{N}$ at infinity. As a consequence, on superhorizon scales the present solution would be consistent with an Einstein-de Sitter cosmology without dark components [40, 41].

The integrated $G(z) / G_{0}$ can be seen in Fig 1 . Also, as a result of the numerical integration, it follows that $\omega$ and $\mu$ are constants as a function of $z$. All these results are consistent with the ones obtained by Moffat \& Toth in 40. On the other hand, it follows from Fig. 1 that if $V_{G}=0$, the behaviour of $G(z) / G_{0}$ does not depend on the value assumed for $H_{0}$. Conversely, if the case with $V_{G} \neq 0$ is considered, different solutions are obtained when different values of $H_{0}$ are considered (see Fig. 1). An explanation for this behavior lies in the fact that, if $V_{G} \neq 0$, a term proportional to $\frac{V_{G}}{G^{2}}$ is added to Eq. 6 .

\section{OBSERVATIONAL CONSTRAINTS}

In this section, we describe the data sets that we use to make the comparison with the predictions of the MOG theory.

\section{A. Cosmic chronometers}

The Cosmic Chronometer (CC) approach is very useful to track the Universe evolution. This method was proposed and implemented in [45] and it allows to determine the Hubble parameter $H(z)$ from the following expression:

$$
H(z)=\frac{-1}{1+z} \frac{d z}{d t}
$$

The method is based on the differential age evolution of old elliptical passive-evolving ${ }^{2}$ galaxies that formed at the same time but are separated by a small redshift interval. In this way, by measuring the age difference of

\footnotetext{
${ }^{2}$ Passive-evolving in the sense that there is no star formation or interaction with other galaxies.
} 


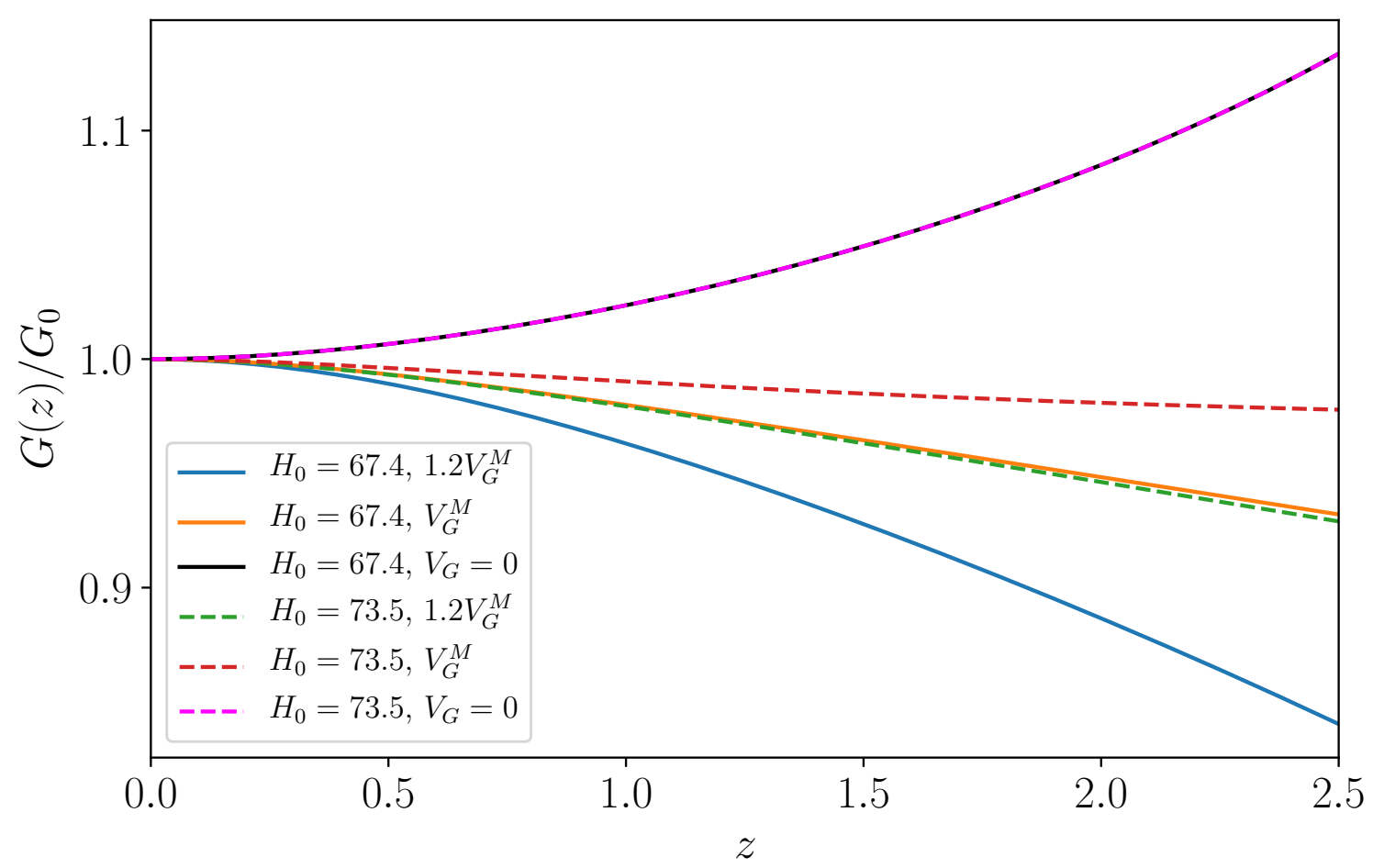

FIG. 1. $\frac{G(z)}{G_{0}}$ for MOG models with fixed values of $H_{0}$ (labeled in the figure in units of $\mathrm{km} \mathrm{s}^{-1} \mathrm{Mpc}^{-1}$ ) and $V_{G}$. $V_{G}^{M}=0.077 \frac{G_{0}^{2}}{t_{0}^{2}}$ is the value suggested in Ref. [40] which also analyses the MOG theory in the cosmological context.

those galaxies, the derivative $\frac{d z}{d t}$ can be measured from the ratio $\frac{\Delta z}{\Delta t}$.

Good candidates for cosmic chronometers are galaxies that have been formed early in the Universe, at high redshift $(z>2-3)$, with large mass $\left(\mathcal{M}_{\text {stars }}>10^{11} \mathcal{M}_{\odot}\right)$, and which have not had any star formation ever since. In this way, if these galaxies are observed at later cosmic time, the age evolution of their stars can be used as a clock synchronized with the evolution of cosmic time. Spectroscopic surveys allow to obtain $d z$ with high accuracy. The main advantage of this method lies in the measurement of relative ages $d t$, which avoids the systematic effects that affect the determination of absolute ages. Moreover, the determination of $d t$ depends only on atomic physics and not on the integrated distance along the line of sight (redshift) which, in turn, is a function of the cosmological model.

In this paper, we consider the most recent and accurate estimates of $H(z)$ acquired through this method (see Table I to test the predictions of the MOG theory. Simon et al. 46] used the GDDS catalogue 47] together with spectroscopic data from other galaxies [48, 49, 50, 51, 52, 53. to obtain 9 determinations of $H(z)$ in the redshift range $0.09 \leq z \leq 1.75$. Stern et al. [54] obtained high quality spectra from red galaxies and used them together with spectra from the SPICES [55] and VVDS [56] catalogues to obtain two measurements of $H(z)$ at $z=$ 0.48 and $z=0.88$. Moresco et al. 57] took a sam- ple of 11,000 massive red galaxies from different catalogues [58, 59, 60, 61, 62, 63, 64, 65, 66, 67, to obtain 8 measurements of $H(z)$ within the redshift range $0.17<z<1.04$. Zhang et al. 68 obtained their results in the redshift interval $0.07 \leq z \leq 0.28$ taking 17,832 red galaxies from the Sloan Digital Sky Survey Data Release Seven (SDSS DR7) 69. Moresco [0] considered spectroscopic data of 29 high redshift galaxies $(z>1.4)$ [71, 72, 73, 74, 75, to obtain estimations of $H(z)$ at redshift $z=1.363$ and $z=1.965$. Moresco et al. [76] used data from the BOSS catalogue [77, 78] to obtain 5 measurements of $H(z)$ in the redshift range $0.38<z<0.48$.

\section{B. Supernovae type Ia}

Type Ia Supernovae (SNe Ia) are among the most energetic events in the Universe. They are quite common, and are observed in different kind of galaxies. The extremely high luminosity of the supernovae makes them easily detectable by surveys. Most importantly, the homogeneity of their spectra and light curves makes them excellent candidates to be considered as standard candles.

The distance modulus of a supernova is given by,

$$
\mu=25+5 \log _{10}\left(d_{L}(z)\right),
$$




\begin{tabular}{|c|c|c|}
\hline$z$ & $H(z)\left(\mathrm{km} \mathrm{s}^{-1} \mathrm{Mpc}^{-1}\right)$ & Reference \\
\hline 0.09 & $69 \pm 12$ & \multirow{9}{*}{ 46] } \\
\hline 0.17 & $83 \pm 8$ & \\
\hline 0.27 & $77 \pm 14$ & \\
\hline 0.4 & $95 \pm 17$ & \\
\hline 0.9 & $117 \pm 23$ & \\
\hline 1.3 & $168 \pm 17$ & \\
\hline 1.43 & $177 \pm 18$ & \\
\hline 1.53 & $140 \pm 14$ & \\
\hline 1.75 & $202 \pm 40$ & \\
\hline 0.48 & $97 \pm 62$ & \multirow[t]{2}{*}{ 54] } \\
\hline 0.88 & $90 \pm 40$ & \\
\hline 0.1791 & $75 \pm 4$ & \multirow{8}{*}{ 57] } \\
\hline 0.1993 & $75 \pm 5$ & \\
\hline 0.3519 & $83 \pm 14$ & \\
\hline 0.5929 & $104 \pm 13$ & \\
\hline 0.6797 & $92 \pm 8$ & \\
\hline 0.7812 & $105 \pm 12$ & \\
\hline 0.8754 & $125 \pm 17$ & \\
\hline 1.037 & $154 \pm 20$ & \\
\hline 0.07 & $69 \pm 19.6$ & \multirow{4}{*}{68} \\
\hline 0.12 & $68.6 \pm 26.2$ & \\
\hline 0.2 & $72.9 \pm 29.6$ & \\
\hline 0.28 & $88.8 \pm 36.6$ & \\
\hline 1.363 & $160 \pm 33.6$ & \multirow[t]{2}{*}{ 70] } \\
\hline 1.965 & $186.5 \pm 50.4$ & \\
\hline 0.3802 & $83 \pm 13.5$ & \multirow{5}{*}{ [76] } \\
\hline 0.4004 & $77 \pm 10.2$ & \\
\hline 0.4247 & $87.1 \pm 11.2$ & \\
\hline 0.4497 & $92.8 \pm 12.9$ & \\
\hline 0.4783 & $80.9 \pm 9$ & \\
\hline
\end{tabular}

TABLE I. $H(z)$ constraints obtained from the cosmic chronometers. The table shows the redshift of the measurement, the mean value and standard deviation of $H(z)$ in units of $\mathrm{km} \mathrm{s}^{-1} \mathrm{Mpc}^{-1}$ and the corresponding reference.

being $d_{L}$ the luminosity distance that depends on the cosmological model and redshift $z$,

$$
d_{L}(z)=(1+z) \int_{0}^{z} \frac{d z^{\prime}}{H\left(z^{\prime}\right)} .
$$

Then, it is possible to compare the theoretical distance modulus of the MOG theory with the modulus obtained from the SNe Ia data.

In this paper, we consider the Pantheon compilation [13] of 1,048 SNe Ia in the redshift interval $0.01<$ $z<2.3$. The observed distance modulus estimator for this compilation is given by

$$
\mu=m_{B}-M+\alpha x_{1}+\beta c+\Delta_{M}+\Delta_{B},
$$

where $\Delta_{M}$ is a distance correction based on the mass of the SNe Ia's host-galaxy, and $\Delta_{B}$ is a distance correction based on predicted biases from simulations. Furthermore, $m_{B}$ is an overall flux normalization, $x_{1}$ and $c$ refer to the deviation from the average light-curve shape and the mean SNe Ia BV color, respectively ${ }^{3}$. Finally, $M$ is the absolute B-band magnitude of a fiducial SNe Ia with $x_{1}=0$ and $c=0$. Parameter $\alpha$ represents the coefficient of the relation between luminosity and stretch; while $\beta$, the coefficient of the relation between luminosity and color.

For the Pantheon data compilation $\Delta_{M}$ is determined by:

$$
\Delta_{M}=\gamma \times\left[1+e^{\left(-\left(m-m_{\text {step }}\right) / \tau\right)}\right]^{-1}
$$

where $\gamma$ stands for a relative offset in luminosity; $m_{\text {step }}$, a mass step for the split; and $\tau$, an exponential transition term in a Fermi function that describes the relative probability of masses being on one side or the other of the split. The last two ( $m_{\text {step }}$ and $\tau$ ) are obtained from different host galaxies samples (technicalities are described in [13]) and $m$ is the host-galaxy mass. Finally, $\alpha, \beta, M$ and $\gamma$ account for nuisance parameters.

\section{BAO}

Before the formation of neutral hydrogen, photons and free electrons were coupled through Thomson scattering, generating accoustic waves in the primordial plasma. After recombination, matter and radiation decouples. The maximum distance the accoustic wave could travel defines a characteristic scale, named the sound horizon at the drag epoch $r_{d}$; this scale is imprinted in the distribution of matter in the Universe. Baryon Accoustic Oscillations (BAO) provide a standard ruler to measure cosmological distances. Different tracers of the underlying matter density field provide probes to measure distances at different redshifts.

Along the line of sight, the BAO signal directly constrains the Hubble constant $H(z)$ at redshift $z$. When measured in a redshift shell, it constrains the angular diameter distance $D_{A}(z)$,

$$
D_{A}(z)=\frac{c}{(1+z)} \int_{0}^{z} \frac{d z^{\prime}}{H\left(z^{\prime}\right)}
$$

To separate $D_{A}(z)$ and $H(z)$, BAO should be measured in the $2 \mathrm{D}$ correlation function, for which extremely large volumes are necessary. If this is not the case, a combination of both quantities can be measured as

$$
D_{V}(z)=\left[(1+z)^{2} D_{A}^{2}(z) \frac{c z}{H(z)}\right]^{1 / 3} .
$$

BAO have been measured with great precision using different observational probes. To measure the BAO

\footnotetext{
3 Parameters $m_{B}, x_{1}$ and $c$ result from the fit of a model of the SNe Ia spectral sequence to the photometric data, details in Ref. [13].
} 


\begin{tabular}{|c|c|c|c|}
\hline$z_{\text {eff }}$ & Value & Observable & Reference \\
\hline 0.16 & $456 \pm 27.0 \mathrm{Mpc}$ & $D_{V}$ & {$[79]$} \\
\hline 0.15 & $(664 \pm 25.0)\left(r_{d} / r_{d}^{f i d}\right) \mathrm{Mpc}$ & $D_{V}$ & {$[15]$} \\
\hline 0.81 & $(1649.5 \pm 66.0)\left(r_{d} / r_{d}^{f i d}\right) \mathrm{Mpc}$ & $D_{A}$ & {$[17]$} \\
\hline 0.38 & $(1512 \pm 33)\left(r_{d} / r_{d}^{f i d}\right) \mathrm{Mpc}$ & $D_{M}$ & \\
0.38 & $(81.2 \pm 3.2)\left(r_{d} / r_{d}^{f i d}\right) \mathrm{km} \mathrm{s}^{-1} \mathrm{Mpc}^{-1}$ & $H$ & \\
0.51 & $(1975 \pm 41)\left(r_{d} / r_{d}^{f i d}\right) \mathrm{Mpc}$ & $D_{M}$ & {$[16]$} \\
0.51 & $(90.9 \pm 3.3)\left(r_{d} / r_{d}^{f i d}\right) \mathrm{km} \mathrm{s}^{-1} \mathrm{Mpc}^{-1}$ & $H$ & \\
0.61 & $(2307 \pm 50)\left(r_{d} / r_{d}^{f i d}\right) \mathrm{Mpc}$ & $D_{M}$ & \\
0.61 & $(99.0 \pm 3.4)\left(r_{d} / r_{d}^{f i d}\right) \mathrm{km} \mathrm{s}^{-1} \mathrm{Mpc}{ }^{-1}$ & $H$ & \\
\hline 0.44 & $(1716 \pm 83.0)\left(r_{d} / r_{d}^{f i d}\right) \mathrm{Mpc}$ & $D_{V}$ & \\
0.6 & $(2221 \pm 101.0)\left(r_{d} / r_{d}^{f i d}\right) \mathrm{Mpc}$ & $D_{V}$ & {$[\underline{80]}$} \\
0.73 & $(2516 \pm 86.0)\left(r_{d} / r_{d}^{f i d}\right) \mathrm{Mpc}$ & $D_{V}$ & \\
\hline 1.52 & $(3843 \pm 147.0)\left(r_{d} / r_{d}^{f i d}\right) \mathrm{Mpc}$ & $D_{V}$ & {$[\underline{81}$} \\
\hline 2.3 & $(1336 \pm 45.7)\left(r_{d} / r_{d}^{f i d}\right) \mathrm{Mpc}$ & $D_{H}$ & {$[\underline{82}$} \\
2.3 & $(5566 \pm 317.2)\left(r_{d} / r_{d}^{f i d}\right) \mathrm{Mpc}$ & $D_{M}$ & \\
\hline 2.4 & $(1327.4 \pm 53.0)\left(r_{d} / r_{d}^{f i d}\right) \mathrm{Mpc}$ & $D_{H}$ & {$[\underline{83}$} \\
2.4 & $(5259.7 \pm 250.5)\left(r_{d} / r_{d}^{f i d}\right) \mathrm{Mpc}$ & $D_{M}$ & \\
\hline
\end{tabular}

TABLE II. Distance constraints from BAO measurements of different observational probes. The table shows the redshift of the measurement, the mean value and standard deviation of the observable, the observable that is measured in each case and the corresponding reference.

scale from the clustering of matter, it is necessary to define a fiducial cosmology. Most of the distance constraints presented in Table II are multiplied by a factor $\left(r_{d} / r_{d}^{f i d}\right)$, which is the ratio between the sound horizon at the drag epoch to the same quantity computed in the fiducial cosmology. We take this ratio as a free parameter in the statistical analysis.

In the following, we describe the observations used in this work. The large-scale correlation function of the $6 \mathrm{dF}$ Galaxy Survey (6dFGS) 79, which is obtained from a K-band selected galaxy subsample with redshifts, determines a value for the isotropic angular diameter distance $D_{V}$ at effective redshift, $z_{\text {eff }}$ of 0.106 . The same quantity at $z_{\text {eff }}=0.15$ is computed in Ross et al. [15, using the main sample of SDSS-DR7 galaxies, with measured redshifts, in combination with a reconstruction method to alleviate the effect of non-linearities on the BAO scale. The first year data release of the Dark Energy Survey [17. provides a measurement of the angular diameter distance $D_{A}$ at $z_{\text {eff }}=0.81$, using the projected two point correlation function of a sample of over 1.3 million galaxies with measured photometric redshifts, distributed over a footprint of $1336 \mathrm{deg}^{2}$. The final galaxy clustering data release of the Baryon Oscillation Spectroscopic Survey [16, provides measurements of the comoving angular diameter distance $D_{M}$ (related with the physical angular diameter distance by $\left.D_{M}(z)=(1+z) D_{A}(z)\right)$ and Hubble parameter $H$ from the BAO method after applying a reconstruc- tion method, for three partially overlapping redshift slices centred at effective redshifts $0.38,0.51$, and 0.61. Using the WiggleZ Dark Energy Survey [80] and a reconstruction method, measurements of $D_{V}$ at effective redshifts of $0.44,0.6$, and 0.73 are provided. With a sample of 147000 quasars from the extended Baryon Oscillation Spectroscopic Survey (eBOSS) [81] distributed over 2044 square degrees with redshifts $0.8<z<2.2$, a measurement of $D_{V}$ at $z_{\text {eff }}=1.52$ is provided. The BAO can be also determined from the flux-transmission correlations in $\operatorname{Ly} \alpha$ forests in the spectra of 157,783 quasars in the redshift range $2.1<z<3.5$ from the Sloan Digital Sky Survey (SDSS) data release 12 (DR12) 82]. Measurements of $D_{M}$ and the Hubble distance (defined as $D_{H}=c / H(z)$ ) at $z_{\text {eff }}=2.33$ are provided. From the cross-correlation of quasars with the Ly $\alpha$-forest flux transmission of the final data release of the SDSS-III [83, a measurement of $D_{M}$ and $D_{H}$ at $z_{\text {eff }}=2.4$ can be obtained.

\section{RESULTS}

In this section, we compare MOG cosmological predictions, explained in Section [I], with the observational data described in Section III. We use $\chi^{2}$ tests to quantify the agreement between the theoretical results and the data. To proceed with the comparison, we define a fiducial model, that will be taken as a reference to analyze the predictions of the MOG models. It is well known that there is a tension between the value of $H_{0}$ obtained from the Cosmic Microwave Background (CMB) [14] and the one inferred from local measurements [84. Therefore, we choose two $\Lambda \mathrm{CDM}$ models with fixed values of $H_{0}$ and the total matter density parameter $\Omega_{m}$ as follows:

- $\Lambda$ CDM Model 1: $H_{0}=67.4 \mathrm{~km} \mathrm{sec}^{-1} \mathrm{Mpc}^{-1}$ and $\Omega_{m}=0.315$; the values obtained by the Planck collaboration using CMB data 14 .

- $\Lambda$ CDM Model 2: $H_{0}=73.5 \mathrm{~km} \mathrm{sec}^{-1} \mathrm{Mpc}^{-1}$ and $\Omega_{m}=0.298$; the values inferred from cepheids and type Ia supernovae in Refs. 84] and [13] respectively.

We perform separate statistical analyses for each data set, for which we test the predictions of the MOG theory for different values of $V_{G}$ and particular values of $H_{0}$ (namely, those of the fiducial models defined above). Results are shown in Tables [II] IV] V] and VI together with the corresponding $\tilde{\chi}_{5 \sigma}^{2}$ value ${ }^{4}$ for the number of data

\footnotetext{
${ }^{4}$ If we assume that the probability distribution function of the reduced $\chi^{2}$ for a given number of data and free parameters is gaussian, its mean value (which corresponds to the maximum probability) is equal to 1 , and a $\sigma$ value for the dispersion can be defined. Furthermore, the $99.99995 \%$ of probability is asigned to the confidence interval $1 \pm\left(\tilde{\chi}_{5 \sigma}^{2}-1\right)$, i.e. the confidence interval at $5 \sigma$.
} 
and free parameters considered in each case. Notice that $V_{G}^{M}=0.077 G_{0}^{2} / t_{0}^{2}$ is the value suggested by [40, who also analyze this theory in a cosmological context.

It follows from Section $\Pi$ II that the theoretical prediction of the MOG theory for the scale factor evolution and its derived quantity $H(z)$ involves no free parameters. On the other hand, eq. (13) shows that the analysis of supernovae data involves several free parameters: the nuisance parameters and the absolute magnitude $M$. In all of the analyses done in this paper, we consider the absolute magnitude $M$ as a free parameter. Regarding the nuisance parameters, we consider two cases: i) fixed values given by the Pantheon compilation (Table III) and ii) the nuisance parameters are allowed to vary (Table IV). The reason for this, is that the nuisance parameters given by the Pantheon compilation were obtained assuming a $\Lambda \mathrm{CDM}$ model for the theoretical predictions of the distance modulus and therefore it is not correct to assume a priori those values when analyzing an alternative cosmological model. Nevertheless, the obtained confidence intervals for the nuisance parameters are consistent with those given by the Pantheon compilation at $1 \sigma$ level. Therefore, the present analysis confirms the robustness of those parameters.

It can be noticed from Table III that only when $V_{G}=0$ is considered, the predictions of the corresponding MOG models are inconsistent with type Ia supernovae data at $5 \sigma$. Conversely, the corresponding predictions of all other MOG models considered in this paper, show agreement with the data within 3- $\sigma$. Accordingly, Fig 2 shows that there is a tiny difference between the theoretical predictions for a MOG model with $V_{G}=V_{G}^{M}, H_{0}=63.4 \mathrm{~km}$ $\mathrm{sec}^{-1} \mathrm{Mpc}^{-1}$ and the respective of $\Lambda \mathrm{CDM}$ model 1, while the model with $V_{G}=0$ fails to predict the behavior of the data. Furthermore, Fig. 3 shows that the difference between the predictions of the $\Lambda \mathrm{CDM}$ model 2 and the MOG model with $V_{G}=V_{G}^{M}$ and $H_{0}=73.5 \mathrm{~km} \mathrm{sec}^{-1}$ $\mathrm{Mpc}^{-1}$, while still small, is greater than the difference between the $\Lambda$ CDM model 1 and the MOG model with $V_{G}=V_{G}^{M}$ and $H_{0}=63.4 \mathrm{~km} \mathrm{sec}^{-1} \mathrm{Mpc}^{-1}$. This might indicate that the MOG theory could be a candidate to alleviate the $H_{0}$ tension.

Similar to the analysis with type Ia supernovae, when cosmic chronometers are considered, results in Table V show that there is no agreement between the theoretical predictions of MOG models with $V_{G}=0$ and the data within 5- $\sigma$. Furthermore, not all MOG models with $V_{G} \neq$ 0 and $H_{0}=73.5 \mathrm{~km} \mathrm{sec}{ }^{-1} \mathrm{Mpc}^{-1}$ are in agreement with cosmic chronometers data within $5 \sigma$; which is the case if $H_{0}=63.4 \mathrm{~km} \mathrm{sec}^{-1} \mathrm{Mpc}^{-1}$ is assumed. On the other hand, Figure 4 shows that the predictions for $H(z)$ change with the selected value of $H_{0}$.

Finally, Table VI shows that only two MOG models with $H_{0}=67.4 \mathrm{~km} \mathrm{sec}^{-1} \mathrm{Mpc}^{-1}$ have theoretical predictions that are in agreement with $\mathrm{BAO}$ data within $2 \sigma$ while for the case $H_{0}=73.5 \mathrm{~km} \mathrm{sec}^{-1} \mathrm{Mpc}^{-1}$ only one model can explain the data within $5 \sigma$. Besides, all other cases considered in this paper show a reduced $\chi^{2}$ value

\begin{tabular}{|c|c|c|c|c|}
\hline \multirow{2}{*}{$V_{G}$} & \multicolumn{2}{|c|}{$M$} & \multicolumn{2}{c|}{$\tilde{\chi}^{2}$} \\
\cline { 2 - 5 } & $H_{0}=67.4$ & $H_{0}=73.5$ & $H_{0}=67.4$ & $H_{0}=73.5$ \\
\hline 0 & $-19.20 \pm 0.0042$ & $-19.01 \pm 0.0042$ & 1.40 & 1.40 \\
$0.6 V_{G}^{M}$ & $-19.33 \pm 0.0042$ & $-19.12 \pm 0.0042$ & 1.08 & 1.12 \\
$0.8 V_{G}^{M}$ & $-19.38 \pm 0.0042$ & $-19.16 \pm 0.0042$ & 1.01 & 1.05 \\
$0.9 V_{G}^{M}$ & $-19.40 \pm 0.0042$ & $-19.18 \pm 0.0042$ & 0.99 & 1.02 \\
$V_{G}^{M}$ & $-19.43 \pm 0.0042$ & $-19.20 \pm 0.0042$ & 0.98 & 1.00 \\
$1.1 V_{G}^{M}$ & $-19.46 \pm 0.0042$ & $-19.22 \pm 0.0042$ & 0.99 & 0.98 \\
$1.2 V_{G}^{M}$ & $-19.49 \pm 0.0042$ & $-19.25 \pm 0.0042$ & 1.03 & 0.98 \\
\hline
\end{tabular}

TABLE III. Results of the statistical analysis performed with Supernovae type Ia data with fixed nuisance parameters. The table shows the fixed values of $H_{0}$ and $V_{G}$ considered in each case, the obtained best fit value and 1- $\sigma$ error for the absolute magnitude $M$ and the corresponding reduced $\chi^{2}$-value. The equivalent $\chi^{2}$ at $5 \sigma$ is $\tilde{\chi}_{5 \sigma}^{2}=1.23$, while for $\Lambda$ CDM fiducial models 1 and 2 we obtain $\tilde{\chi}_{\Lambda \mathrm{CDM}}^{2}=0.98 . V_{G}^{M}$ is the value suggested in Ref. [40] which also analyses the MOG theory in the cosmological context.

\begin{tabular}{|c|c|c|c|c|c|}
\hline$H_{0}$ & $\alpha$ & $\beta$ & $\gamma$ & $M$ & $\tilde{\chi}^{2}$ \\
\hline 67.4 & - & - & - & $-19.43 \pm 0.004$ & 0.98 \\
73.5 & - & - & - & $-19.20 \pm 0.004$ & 1.00 \\
67.4 & $0.158 \pm 0.005$ & $3.03 \pm 0.05$ & $0.051 \pm 0.009$ & $-19.43 \pm 0.007$ & 0.99 \\
73.5 & $0.156 \pm 0.005$ & $3.00 \pm 0.05$ & $0.056 \pm 0.009$ & $-19.19 \pm 0.007$ & 1.01 \\
\hline Pantheon & $0.154 \pm 0.006$ & $3.02 \pm 0.06$ & $0.053 \pm 0.009$ & & \\
\hline
\end{tabular}

TABLE IV. Results of the statistical analysis performed with Supernovae type Ia data. In all cases $V_{G}=V_{G}^{M}$. The first column shows the fixed values of $H_{0}$ considered in each case. The first two entries show the results for fixed nuisance parameters given by the Pantheon compilation (also shown in Table III). The third and fourth entries show the results for the case where the nuisance parameters are allowed to vary. The equivalent $\chi^{2}$ at $5 \sigma$ for the latter is $\tilde{\chi}_{5 \sigma}^{2}=1.23$. The last entry shows the values of the nuisance parameters obtained by the Pantheon compilation.

beyond the customary $5-\sigma$ equivalent $\tilde{\chi}_{5 \sigma}^{2}$. This behavior can also be appreciated in Figs. 5 and 6 . Therefore, it should be noted that BAO data, which comprise several independent data sets, as described in Section IIIC, provide a useful tool to validate the predictions of the different MOG models analyzed in this paper. On the contrary, the predictions of the MOG models for type Ia supernovae data are very similar to the $\Lambda$ CDM fiducial model's ones, provided $V_{G} \neq 0$. Regarding the cosmic chronometers, even though the predictions for the MOG models do not match the $\Lambda \mathrm{CDM}$ fiducial model's one, the large error bars in Fig. 4 prevent this data set to be more conclusive when testing the MOG models. 

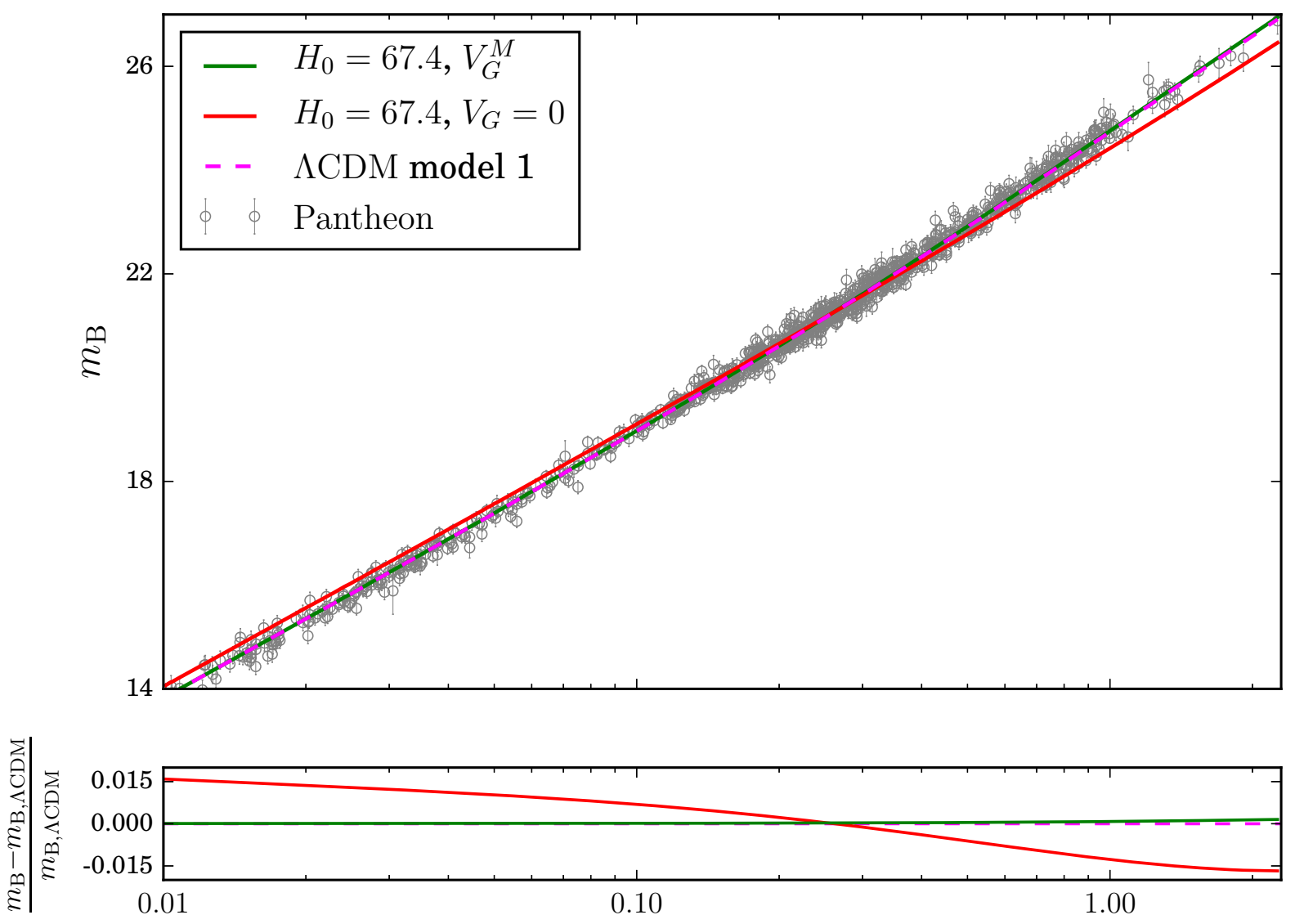

0.10

1.00

FIG. 2. Upper panel: $m_{\mathrm{B}}$ for the fiducial $\Lambda$ CDM model 1 and MOG models considering $H_{0}=67.4 \mathrm{~km} \mathrm{sec}^{-1} \mathrm{Mpc}^{-1}$ and fixed values of $V_{G}$. The data of the Pantheon compilation are shown in gray circles. The $\Lambda$ CDM fiducial model 1 and the MOG model with $V_{G}=V_{G}^{M}$ are in agreement with the data within $5 \sigma$ while for the MOG model with $V_{G}=0$ the obtained reduced $\chi^{2}$ value falls beyond the $5-\sigma$ equivalent. Lower panel: relative differences between the $\Lambda$ CDM model 1 and the MOG theory for different values for $H_{0}$ and $V_{G}$.

\section{SUMMARY AND CONCLUSIONS}

In this paper we have analyzed the MOG theory in the cosmological context. We considered recent data sets from SNe Ia, BAO and CC to perform $\chi^{2}$ tests. We considered models with fixed values of $H_{0}$ and $V_{G}$, the selfinteraction potential of the scalar field that represents the gravitational constant in this theory.

Our results show that none of the predictions of the MOG theory considered here with $V_{G}=0$ is in agreement with the data, which is also consistent with previous results 40, 41, 43, 44. Regarding the $\mathrm{SNe}$ Ia data, we have verified that for different non-zero values of $V_{G}$, the difference between the predictions of the MOG theory is very tiny (in fact, adding more MOG models to Figs 2 and 3 would have been confusing due to the superposition of curves). Conversely, in the case of the CC data, the predictions vary with the value of $V_{G}$ and $H_{0}$, but the large error bars prevent it from being possible to discard more models with this data set. Furthermore, we have also shown that there is agreement between all $\mathrm{SNe} \mathrm{Ia}$ and all but one $\mathrm{CC}$ data and the predictions of the MOG theory. The result is completely different in the case of the statistical analysis with the BAO data set, in which only three models are consistent with the data within $5 \sigma$. Furthermore, it should be stressed that the statistical significance of these latter results is greater when $H_{0}=64.7 \mathrm{~km} \mathrm{sec}^{-1} \mathrm{Mpc}^{-1}$ is considered.

On the other hand, the present analysis confirms that the nuisance parameters obtained by the Pantheon compilation assuming the $\Lambda \mathrm{CDM}$ model are also valid for the MOG theory and therefore it is likely for them to be accurate for other non-standard cosmological models.

In summary, we have analysed the predictions of MOG theory for different values of the Hubble constant and of the self-interaction potential of the scalar field, and compared them to cosmological data of distinct nature, at different redshifts. We have found that most of the 

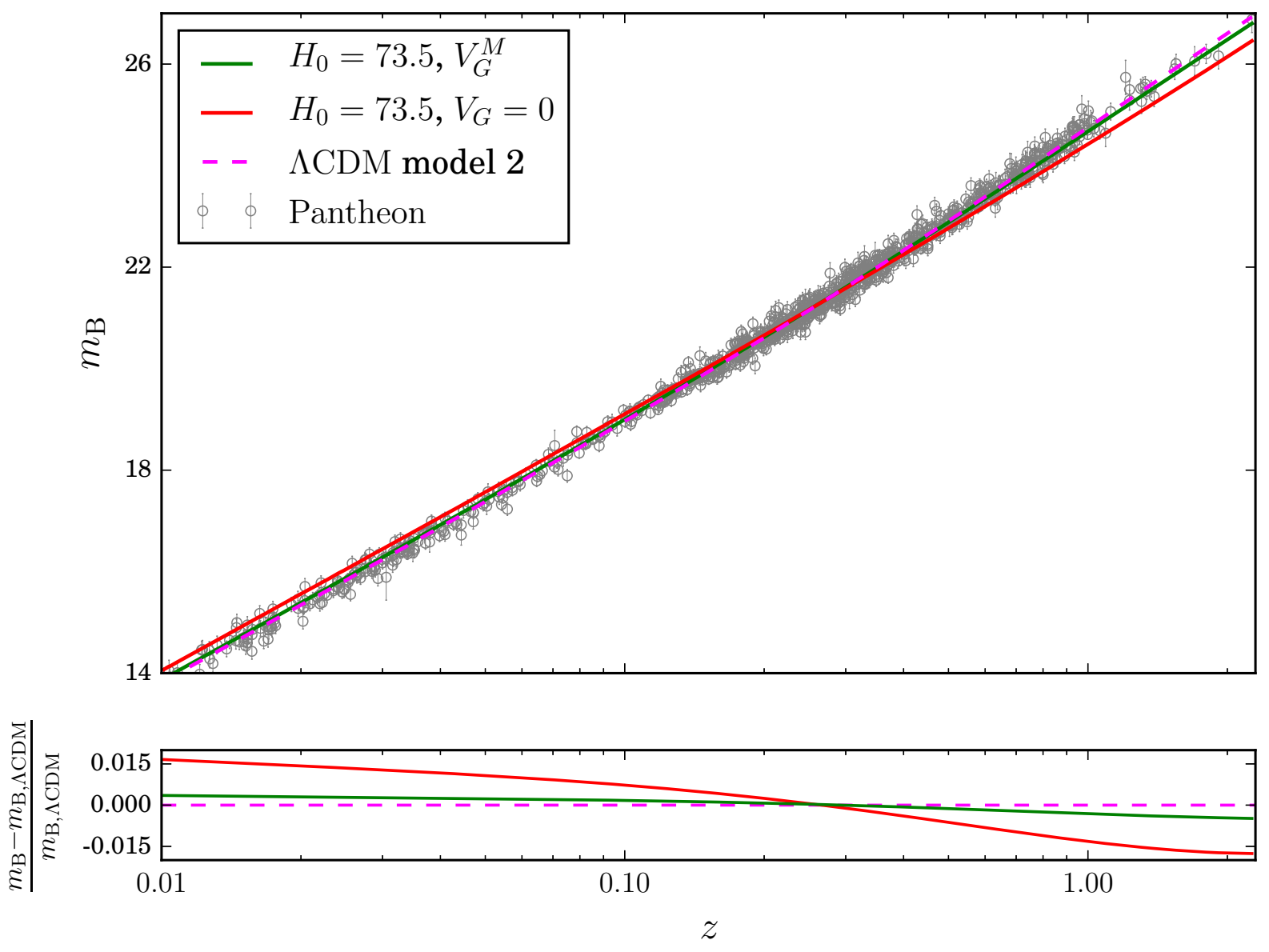

FIG. 3. Upper panel: $m_{\mathrm{B}}$ for the fiducial $\Lambda$ CDM model 2 and MOG models considering $H_{0}=73.5 \mathrm{~km} \mathrm{sec}^{-1} \mathrm{Mpc}{ }^{-1}$ and fixed values of $V_{G}$. The data of the Pantheon compilation are shown in gray circles. The $\Lambda$ CDM fiducial model 2 and the MOG model with $V_{G}=V_{G}^{M}$ are in agreement with the data within $5 \sigma$ while for the MOG model with $V_{G}=0$ the obtained reduced $\chi^{2}$ value falls beyond the 5- $\sigma$ equivalent. Lower panel: relative differences between $\Lambda$ CDM model 2 and the MOG theory for different values for $H_{0}$ and $V_{G}$.

studied cases are ruled out by BAO data set, although there are still some values for which the MOG theory cannot be ruled out for any of the data sets considered in this work. This might mean that MOG theory is still a valid alternative to the dark sector of the Universe.

\section{ACKNOWLEDGEMENTS}

The authors are supported by the National Agency for the Promotion of Science and Technology (ANPCYT) of Argentina grant PICT-2016-0081; and grants G140 and G157 from UNLP. S.L. acknowledges support from grant UBACYT 20020170100129BA
[1] J. H. Oort, Bulletin of the Astronomical Institutes of the Netherlands 6, 249 (1932).

[2] F. Zwicky, Astrophysical Journal 86, 217 (1937).

[3] V. C. Rubin, J. Ford, W. K., and N. Thonnard, Astrophysical Journal 1 225, L107 (1978).

[4] V. C. Rubin, J. Ford, W. K., and N. Thonnard, Astrophysical Journal 238, 471 (1980).

[5] J. A. Tyson, G. P. Kochanski, and I. P. Dell'Antonio, Astrophys. J. Lett. 498, L107 (1998), astro-ph/9801193.
[6] M. Hasselfield, M. Hilton, T. A. Marriage, G. E. Addison, L. F. Barrientos, N. Battaglia, E. S. Battistelli, J. R. Bond, D. Crichton, S. Das, et al., Journal of Cosmology and Astroparticle Physics 7, 008 (2013), 1301.0816.

[7] K. Osato, S. Flender, D. Nagai, M. Shirasaki, and N. Yoshida, Mon. Not. Roy. Astron.Soc. 475, 532 (2018), 1706.08972.

[8] Planck Collaboration, P. A. R. Ade, N. Aghanim, M. Arnaud, M. Ashdown, J. Aumont, C. Baccigalupi, A. J. 


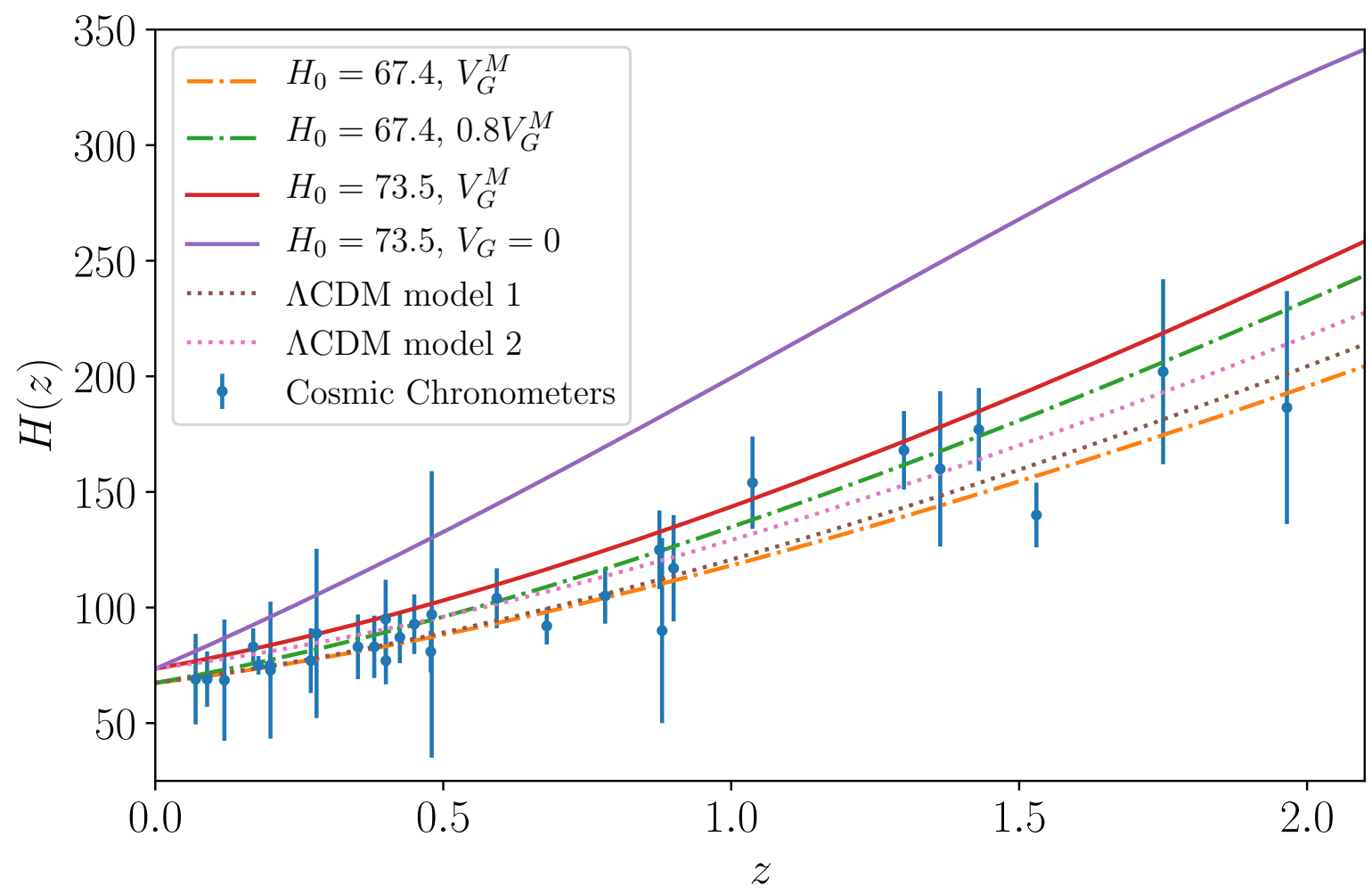

FIG. 4. $H(z)$ for the $\Lambda$ CDM fiducial models 1 and 2 and the MOG models considering different values for $H_{0}$ and $V_{G}$. The observational data from cosmic chronometers are shown in blue dots. The obtained $\chi^{2}$ values for the MOG model with $H_{0}=73.5 \mathrm{~km} \mathrm{sec}^{-1} \mathrm{Mpc}^{-1}$ and $V_{G}=0$ falls beyond the 5- $\sigma$ equivalent while all other models shown in this figure are consistent with the data within $5 \sigma$.

\begin{tabular}{|c|c|c|}
\hline \multirow{2}{*}{$V_{G}$} & \multicolumn{2}{|c|}{$\tilde{\chi}^{2}$} \\
\cline { 2 - 3 } & $H_{0}=67.4$ & $H_{0}=73.5$ \\
\hline 0 & 7.87 & 13.64 \\
$0.6 V_{G}^{M}$ & 1.89 & 5.57 \\
$0.8 V_{G}^{M}$ & 0.78 & 3.41 \\
$0.9 V_{G}^{M}$ & 0.52 & 2.49 \\
$V_{G}^{M}$ & 0.53 & 1.71 \\
$1.1 V_{G}^{M}$ & 0.94 & 1.10 \\
$1.2 V_{G}^{M}$ & 1.94 & 0.71 \\
\hline
\end{tabular}

TABLE V. Results of the statistical analysis performed with cosmic chronometers. The table shows the fixed values of $H_{0}$ and $V_{g}$ considered in each case, and the reduced $\chi^{2}$-value. The equivalent $\chi^{2}$ at $5 \sigma$ is $\tilde{\chi}_{5 \sigma}^{2}=2.79$ while for the $\Lambda \mathrm{CDM}$ fiducial models we obtain: $\tilde{\chi}_{\Lambda \mathrm{CDM} 1}^{2}=0.5$ and $\tilde{\chi}_{\Lambda \mathrm{CDM} 2}^{2}=0.74$.

Banday, R. B. Barreiro, J. G. Bartlett, et al., Astronomy and Astrophysics 594, A24 (2016), 1502.01597.

[9] T. Sakamoto, M. Chiba, and T. C. Beers, Astronomy and Astrophysics 397, 899 (2003), astro-ph/0210508.

[10] X. X. Xue, H. W. Rix, G. Zhao, P. Re Fiorentin, T. Naab, M. Steinmetz, F. C. van den Bosch, T. C. Beers, Y. S.

\begin{tabular}{|c|c|c|c|c|}
\hline \multirow{2}{*}{$V_{G}$} & \multicolumn{2}{|c|}{$r_{d} / r_{d}^{\mathrm{fid}}$} & \multicolumn{2}{c|}{$\tilde{\chi}^{2}$} \\
\cline { 2 - 5 } & $H_{0}=67.4$ & $H_{0}=73.5$ & $H_{0}=67.4$ & $H_{0}=73.5$ \\
\hline 0 & $0.943 \pm 0.008$ & $0.947 \pm 0.008$ & 46.72 & 84.82 \\
$0.6 V_{G}^{M}$ & $0.949 \pm 0.008$ & $0.924 \pm 0.008$ & 11.72 & 37.47 \\
$0.8 V_{G}^{M}$ & $0.967 \pm 0.008$ & $0.925 \pm 0.008$ & 4.02 & 23.39 \\
$0.9 V_{G}^{M}$ & $0.982 \pm 0.008$ & $0.928 \pm 0.008$ & 1.58 & 17.08 \\
$V_{G}^{M}$ & $1.007 \pm 0.008$ & $0.934 \pm 0.008$ & 0.9 & 11.43 \\
$1.1 V_{G}^{M}$ & $1.047 \pm 0.008$ & $0.943 \pm 0.008$ & 4.55 & 6.70 \\
$1.2 V_{G}^{M}$ & $1.138 \pm 0.010$ & $0.956 \pm 0.008$ & 28.12 & 3.39 \\
\hline
\end{tabular}

TABLE VI. Results of the statistical analysis performed with BAO data. The table shows the fixed values of $H_{0}$ and $V_{G}$ considered in each case, and the corresponding reduced $\chi^{2}$ value. The equivalent $\chi^{2}$ at $5 \sigma$ is $\tilde{\chi}_{5 \sigma}^{2}=3.73$ while for the $\Lambda \mathrm{CDM}$ fiducial models we obtain $\tilde{\chi}_{\Lambda \mathrm{CDM} 1}^{2}=0.94$ and $\tilde{\chi}_{\Lambda \mathrm{CDM} 2}^{2}=3.68$.

Lee, E. F. Bell, et al., Astrophysical Journal 684, 1143 (2008), 0801.1232.

[11] P. R. Kafle, S. Sharma, G. F. Lewis, and J. BlandHawthorn, Astrophysical Journal 794, 59 (2014), 
^CDM model 1
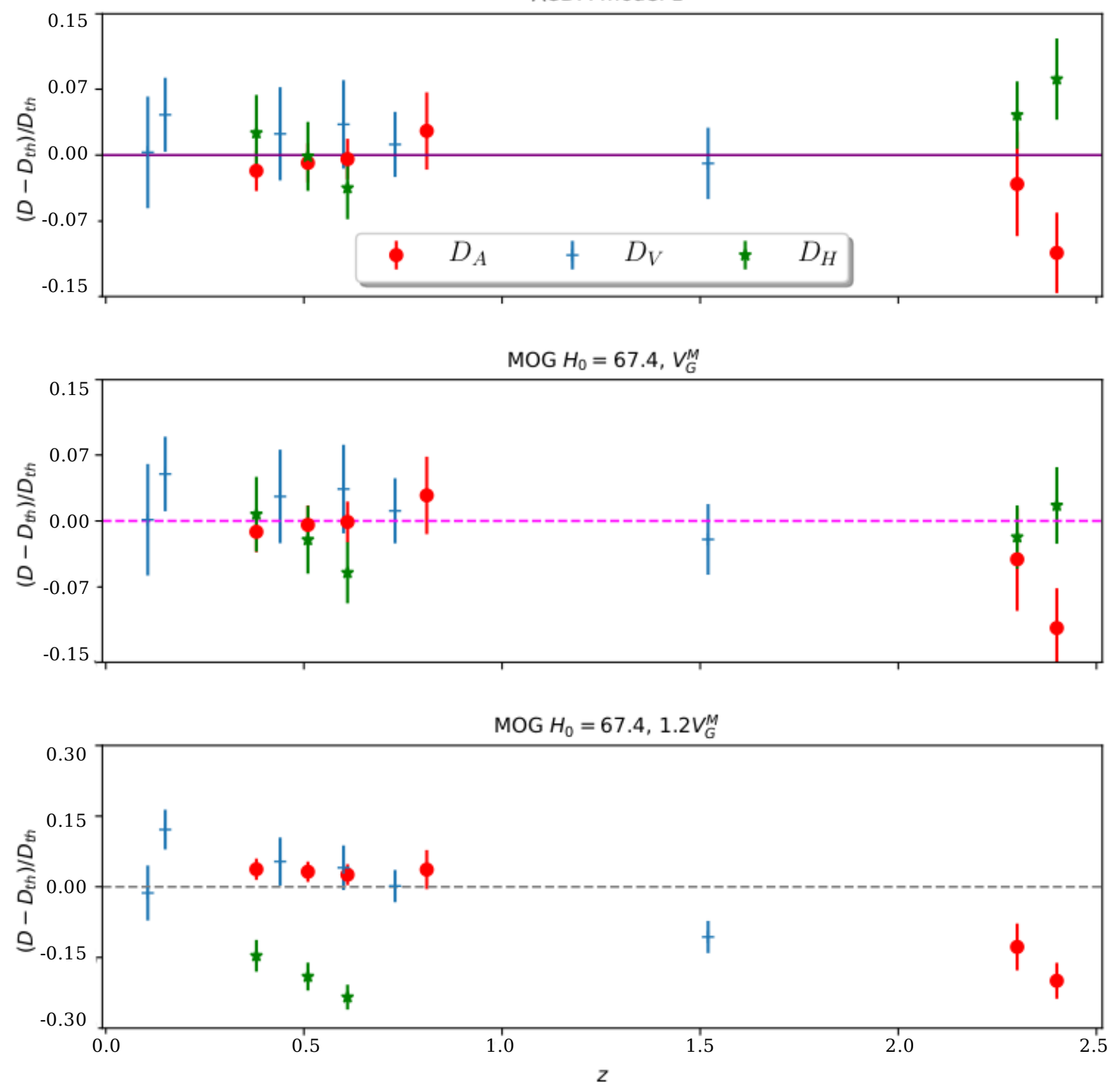

FIG. 5. Relative differences between the observable distance $\left(D_{A}, D_{V}\right.$, or $\left.D_{H}\right)$ and the corresponding theoretical prediction for the $\Lambda$ CDM model 1 and the MOG models with different values for $H_{0}$ and $V_{G}$. The fiducial $\Lambda$ CDM model 1 is shown in the upper panel and $\tilde{\chi}_{\Lambda \mathrm{CDM}}^{2}=0.94$.

1408.1787.

[12] F. Iocco, M. Pato, and G. Bertone, Nature Physics 11, 245 (2015), 1502.03821.

[13] D. M. Scolnic, D. O. Jones, A. Rest, Y. C. Pan, R. Chornock, R. J. Foley, M. E. Huber, R. Kessler, G. Narayan, A. G. Riess, et al., Astrophysical Journal 859, 101 (2018), 1710.00845.

[14] Planck Collaboration, N. Aghanim, Y. Akrami, M. Ashdown, J. Aumont, C. Baccigalupi, M. Ballardini, A. J. Banday, R. B. Barreiro, N. Bartolo, et al., arXiv e-prints arXiv:1807.06209 (2018), 1807.06209.

[15] A. J. Ross, L. Samushia, C. Howlett, W. J. Percival, A. Burden, and M. Manera, Monthly Notices of the Royal Astronomical Society 449, 835847 (2015), ISSN 0035-
8711, URL http://dx.doi.org/10.1093/mnras/stv154

[16] S. Alam, M. Ata, S. Bailey, F. Beutler, D. Bizyaev, J. A. Blazek, A. S. Bolton, J. R. Brownstein, A. Burden, C.-H. Chuang, et al., Monthly Notices of the Royal Astronomical Society 470, 26172652 (2017), ISSN 1365-2966, URL http://dx.doi.org/10.1093/mnras/stx721

[17] T. M. C. Abbott, F. B. Abdalla, A. Alarcon, S. Allam, F. Andrade-Oliveira, J. Annis, S. Avila, M. Banerji, N. Banik, K. Bechtol, et al., Monthly Notices of the Royal Astronomical Society 483, 48664883 (2018), ISSN 1365-2966, URL http://dx.doi.org/10.1093/mnras/ sty3351.

[18] S. E. Nuza, A. G. Sánchez, F. Prada, A. Klypin, D. J. Schlegel, S. Gottlöber, A. D. Montero-Dorta, M. Man- 
NCDM model 2
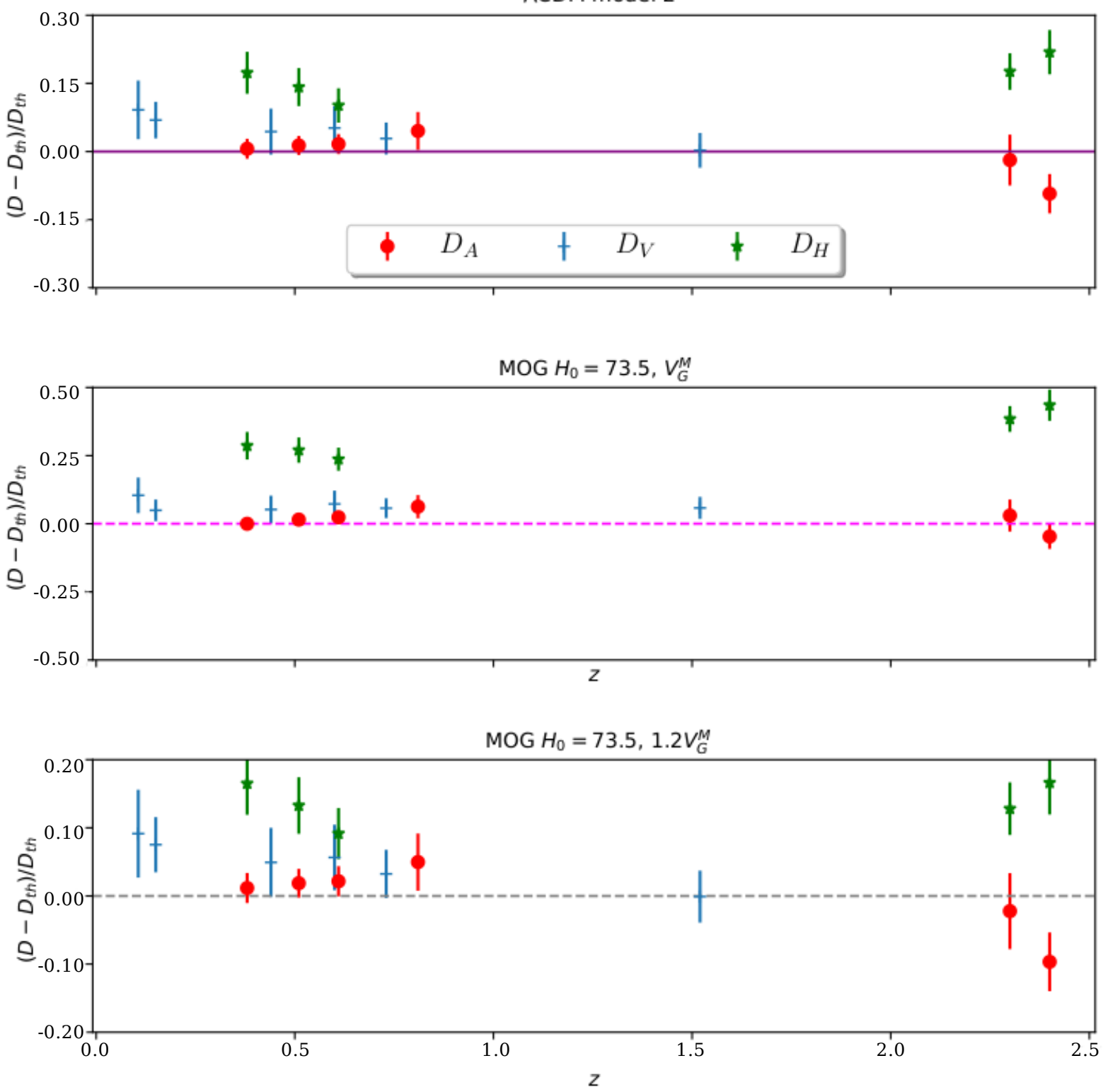

FIG. 6. Relative differences between the observable distance $\left(D_{A}, D_{V}\right.$, or $\left.D_{H}\right)$ and the corresponding theoretical prediction for the $\Lambda$ CDM model 2 and the MOG models with different values for $H_{0}$ and $V_{G}$. The fiducial $\Lambda$ CDM model is shown in the upper panel and $\tilde{\chi}_{\Lambda \mathrm{CDM}}^{2}=3.68$.

era, C. K. McBride, A. J. Ross, et al., Mon. Not. Roy. Astron.Soc. 432, 743 (2013), 1202.6057.

[19] M. Schumann, Journal of Physics G Nuclear Physics 46, 103003 (2019), 1903.03026.

[20] M. Milgrom, Astrophysical Journal 270, 365 (1983).

[21] J. D. Bekenstein, Phys. Rev. D 70, 083509 (2004), astro$\mathrm{ph} / 0403694$.

[22] D. Clowe, M. Bradač, A. H. Gonzalez, M. Markevitch, S. W. Randall, C. Jones, and D. Zaritsky, Astrophysical Journal 1 648, L109 (2006), astro-ph/0608407.

[23] J. W. Moffat, Journal of Cosmology and Gravitation 3, 004 (2006), gr-qc/0506021.

[24] J. W. Moffat and V. T. Toth, Astrophysical Journal 680, 1158-1161 (2008), 0708.1935.
[25] J. W. Moffat and S. Rahvar, MNRAS 441, 3724 (2014), 1309.5077

[26] J. W. Moffat and M. H. Zhoolideh Haghighi, European Physical Journal Plus 132, 417 (2017).

[27] J. W. Moffat and S. Rahvar, MNRAS 436, 1439 (2013), 1306.6383.

[28] M. H. Zhoolideh Haghighi and S. Rahvar, MNRAS 468, 4048 (2017), 1609.07851.

[29] C. Negrelli, M. Benito, S. Landau, F. Iocco, and L. Kraiselburd, Phys. Rev. D 98, 104061 (2018), 1810.07200

[30] D. Clowe, M. Bradač, A. H. Gonzalez, M. Markevitch, S. W. Randall, C. Jones, and D. Zaritsky, Astrophysical Journal Letters 648, L109 (2006), astro-ph/0608407. 
[31] J. R. Brownstein and J. W. Moffat, MNRAS 382, 29 (2007), astro-ph/0702146.

[32] N. S. Israel and J. W. Moffat, ArXiv e-prints (2016), 1606.09128 .

[33] T. M. Nieuwenhuizen, A. Morandi, and M. Limousin, Mon. Not. Roy. Astron.Soc. (2018), 1802.04891.

[34] S. Boran, S. Desai, E. O. Kahya, and R. P. Woodard, Phys. Rev. D 97, 041501 (2018), 1710.06168.

[35] M. A. Green, J. W. Moffat, and V. T. Toth, Physics Letters B 780, 300 (2018), 1710.11177.

[36] B. P. Schmidt, N. B. Suntzeff, M. M. Phillips, R. A. Schommer, A. Clocchiatti, R. P. Kirshner, P. Garnavich, P. Challis, B. Leibundgut, J. Spyromilio, et al., Astrophysical Journal 507, 46 (1998), astro-ph/9805200.

[37] S. Tsujikawa, Dark Energy: Investigation and Modeling, vol. 370 of Astrophysics and Space Science Library (2011).

[38] A. De Felice and S. Tsujikawa, Living Reviews in Relativity 13, 3 (2010), 1002.4928.

[39] J. W. Moffat and V. T. Toth, Classical and Quantum Gravity 26, 085002 (2009), 0712.1796.

[40] J. W. Moffat and V. T. Toth, arXiv e-prints (2007), 0710.0364 .

[41] J. Moffat and V. Toth, Galaxies 1, 65 (2013).

[42] V. T. Toth, arXiv e-prints arXiv:1011.5174 (2010), 1011.5174 .

[43] S. Jamali and M. Roshan, European Physical Journal C 76, 490 (2016), 1608.06251.

[44] S. Jamali, M. Roshan, and L. Amendola, arXiv e-prints arXiv:1811.04445 (2018), 1811.04445.

[45] R. Jimenez and A. Loeb, Astrophysical Journal 573, 37 (2002), astro-ph/0106145.

[46] J. Simon, L. Verde, and R. Jimenez, Phys. Rev. D 71, 123001 (2005), astro-ph/0412269.

[47] R. G. Abraham, K. Glazebrook, P. J. McCarthy, D. Crampton, R. Murowinski, I. Jørgensen, K. Roth, I. M. Hook, S. Savaglio, H.-W. Chen, et al., Astronomical Journal 127, 2455 (2004), astro-ph/0402436.

[48] J. Dunlop, J. Peacock, H. Spinrad, A. Dey, R. Jimenez, D. Stern, and R. Windhorst, Nature (London) 381, 581 (1996).

[49] L. A. Nolan, J. S. Dunlop, R. Jimenez, and A. F. Heavens, Mon. Not. R. Astron. Soc. 341, 464 (2003), astro$\mathrm{ph} / 0103450$.

[50] H. Spinrad, A. Dey, D. Stern, J. Dunlop, J. Peacock, R. Jimenez, and R. Windhorst, Astrophysical Journal 484, 581 (1997), astro-ph/9702233.

[51] T. Treu, M. Stiavelli, S. Casertano, P. Møller, and G. Bertin, Mon. Not. R. Astron. Soc. 308, 1037 (1999), astro-ph/9904327.

[52] T. Treu, M. Stiavelli, P. Møller, S. Casertano, and G. Bertin, Mon. Not. R. Astron. Soc. 326, 221 (2001), astro-ph/0104177.

[53] T. Treu, M. Stiavelli, S. Casertano, P. Møller, and G. Bertin, Astrophysical Journal l 564, L13 (2002), astro-ph/0111504.

[54] D. Stern, R. Jimenez, L. Verde, M. Kamionkowski, and S. A. Stanford, JCAP 2, 008 (2010), 0907.3149.

[55] D. Stern, A. Connolly, P. Eisenhardt, R. Elston, B. Holden, P. Rosati, S. A. Stanford, H. Spinrad, P. Tozzi, and K. Wu, in Deep Fields, edited by S. Cristiani, A. Renzini, and R. E. Williams (2001), p. 76, astro$\mathrm{ph} / 0012146$.
[56] O. Le Fèvre, G. Vettolani, B. Garilli, L. Tresse, D. Bottini, V. Le Brun, D. Maccagni, J. P. Picat, R. Scaramella, M. Scodeggio, et al., Astronomy \& Astrophysics 439, 845 (2005), astro-ph/0409133.

[57] M. Moresco, A. Cimatti, R. Jimenez, L. Pozzetti, G. Zamorani, M. Bolzonella, J. Dunlop, F. Lamareille, M. Mignoli, H. Pearce, et al., JCAP 8, 006 (2012), 1201.3609

[58] A. Cimatti, L. Pozzetti, M. Mignoli, E. Daddi, N. Menci, F. Poli, A. Fontana, A. Renzini, G. Zamorani, T. Broadhurst, et al., Astronomy \& Astrophysics 391, L1 (2002), astro-ph/0207191.

[59] R. Demarco, R. Gobat, P. Rosati, C. Lidman, A. Rettura, M. Nonino, A. van der Wel, M. J. Jee, J. P. Blakeslee, H. C. Ford, et al., Astrophysical Journal 725, 1252 (2010), 1009.3986.

[60] D. J. Eisenstein, J. Annis, J. E. Gunn, A. S. Szalay, A. J. Connolly, R. C. Nichol, N. A. Bahcall, M. Bernardi, S. Burles, F. J. Castander, et al., Astronomical Journal 122, 2267 (2001), astro-ph/0108153.

[61] D. Le Borgne, R. Abraham, K. Daniel, P. J. McCarthy, K. Glazebrook, S. Savaglio, D. Crampton, S. Juneau, R. G. Carlberg, H.-W. Chen, et al., Astrophysical Journal 642, 48 (2006), astro-ph/0503401.

[62] S. J. Lilly, V. Le Brun, C. Maier, V. Mainieri, M. Mignoli, M. Scodeggio, G. Zamorani, M. Carollo, T. Contini, J.P. Kneib, et al., Astrophysical Journal Supplement Series 184, 218 (2009).

[63] M. Onodera, E. Daddi, R. Gobat, M. Cappellari, N. Arimoto, A. Renzini, Y. Yamada, H. J. McCracken, C. Mancini, P. Capak, et al., Astrophysical Journal 1 715, L6 (2010), 1004.2120.

[64] P. Rosati, P. Tozzi, R. Gobat, J. S. Santos, M. Nonino, R. Demarco, C. Lidman, C. R. Mullis, V. Strazzullo, H. Böhringer, et al., Astronomy \& Astrophysics 508, 583 (2009), 0910.1716.

[65] D. Stern, R. Jimenez, L. Verde, M. Kamionkowski, and S. A. Stanford, JCAP 2010, 008 (2010), 0907.3149.

[66] M. A. Strauss, D. H. Weinberg, R. H. Lupton, V. K. Narayanan, J. Annis, M. Bernardi, M. Blanton, S. Burles, A. J. Connolly, J. Dalcanton, et al., Astronomical Journal 124, 1810 (2002), astro-ph/0206225.

[67] E. Vanzella, S. Cristiani, M. Dickinson, M. Giavalisco, H. Kuntschner, J. Haase, M. Nonino, P. Rosati, C. Cesarsky, H. C. Ferguson, et al., Astronomy \& Astrophysics 478, 83 (2008), 0711.0850.

[68] C. Zhang, H. Zhang, S. Yuan, S. Liu, T.-J. Zhang, and Y.-C. Sun, Research in Astronomy and Astrophysics 14, 1221-1233 (2014), 1207.4541.

[69] K. N. Abazajian, J. K. Adelman-McCarthy, M. A. Agüeros, S. S. Allam, C. Allende Prieto, D. An, K. S. J. Anderson, S. F. Anderson, J. Annis, N. A. Bahcall, et al., Astrophysical Journal Supplement Series 182, 543 (2009), 0812.0649.

[70] M. Moresco, Mon. Not. R. Astron. Soc. 450, L16 (2015), 1503.01116 .

[71] R. Gobat, V. Strazzullo, E. Daddi, M. Onodera, M. Carollo, A. Renzini, A. Finoguenov, A. Cimatti, C. Scarlata, and N. Arimoto, Astrophysical Journal 776, 9 (2013), 1305.3576 .

[72] M. Kriek, P. G. van Dokkum, I. Labbé, M. Franx, G. D. Illingworth, D. Marchesini, and R. F. Quadri, Astrophysical Journal 700, 221 (2009), 0905.1692. 
[73] J. K. Krogager, A. W. Zirm, S. Toft, A. Man, and G. Brammer, Astrophysical Journal 797, 17 (2014), 1309.6316.

[74] M. Onodera, A. Renzini, M. Carollo, M. Cappellari, C. Mancini, V. Strazzullo, E. Daddi, N. Arimoto, R. Gobat, Y. Yamada, et al., Astrophysical Journal 755, 26 (2012), 1206.1540.

[75] P. Saracco, M. Longhetti, P. Severgnini, R. Della Ceca, V. Braito, F. Mannucci, R. Bender, N. Drory, G. Feulner, U. Hopp, et al., Mon. Not. R. Astron. Soc. 357, L40 (2005), astro-ph/0412020.

[76] M. Moresco, L. Pozzetti, A. Cimatti, R. Jimenez, C. Maraston, L. Verde, D. Thomas, A. Citro, R. Tojeiro, and D. Wilkinson, JCAP 5, 014 (2016), 1601.01701.

[77] K. S. Dawson, D. J. Schlegel, C. P. Ahn, S. F. Anderson, É. Aubourg, S. Bailey, R. H. Barkhouser, J. E. Bautista, A. r. Beifiori, A. A. Berlind, et al., Astronomical Journal 145, 10 (2013), 1208.0022.

[78] D. J. Eisenstein, D. H. Weinberg, E. Agol, H. Aihara, C. Allende Prieto, S. F. Anderson, J. A. Arns, É. Aubourg, S. Bailey, E. Balbinot, et al., Astronomical Journal 142, 72 (2011), 1101.1529.

[79] F. Beutler, C. Blake, M. Colless, D. H. Jones, L. StaveleySmith, L. Campbell, Q. Parker, W. Saunders, and F. Watson, Mon. Not. R. Astron. Soc. 416, 3017 (2011), 1106.3366 .
[80] E. A. Kazin, J. Koda, C. Blake, N. Padmanabhan, S. Brough, M. Colless, C. Contreras, W. Couch, S. Croom, D. J. Croton, et al., Monthly Notices of the Royal Astronomical Society 441, 35243542 (2014), ISSN 0035-8711, URL http://dx.doi.org/10.1093/mnras/ stu778

[81] M. Ata, F. Baumgarten, J. Bautista, F. Beutler, D. Bizyaev, M. R. Blanton, J. A. Blazek, A. S. Bolton, J. Brinkmann, J. R. Brownstein, et al., Monthly Notices of the Royal Astronomical Society 473, 47734794 (2017), ISSN 1365-2966, URL http://dx.doi.org/10. $1093 / \mathrm{mnras} / \mathrm{stx} 2630$

[82] J. E. Bautista, N. G. Busca, J. Guy, J. Rich, M. Blomqvist, H. du Mas des Bourboux, M. M. Pieri, A. Font-Ribera, S. Bailey, T. Delubac, et al., Astronomy \& Astrophysics 603, A12 (2017), ISSN 1432-0746, URL http://dx.doi.org/10.1051/0004-6361/201730533

[83] H. du Mas des Bourboux, J.-M. Le Goff, M. Blomqvist, N. G. Busca, J. Guy, J. Rich, C. Yche, J. E. Bautista, . Burtin, K. S. Dawson, et al., Astronomy \& Astrophysics 608, A130 (2017), ISSN 1432-0746, URL http: //dx.doi.org/10.1051/0004-6361/201731731

[84] A. G. Riess, S. Casertano, W. Yuan, L. Macri, J. Anderson, J. W. MacKenty, J. B. Bowers, K. I. Clubb, A. V. Filippenko, D. O. Jones, et al., Astrophysical Journal 855, 136 (2018), 1801.01120. 